Meta

Journal des traducteurs

Translators' Journal

\title{
De la localisation à la délocalisation - le facteur local en traduction
}

\section{Nicolas Froeliger et Jean-René Ladmiral}

Volume 55, numéro 4, décembre 2010

De la localisation à la délocalisation - le facteur local en traduction

From Localization to Delocalization - The Local Factor in Translation

URI : https://id.erudit.org/iderudit/045681ar

DOI : https://doi.org/10.7202/045681ar

Aller au sommaire du numéro

Éditeur(s)

Les Presses de l'Université de Montréal

ISSN

0026-0452 (imprimé)

1492-1421 (numérique)

Découvrir la revue

Citer ce document

Froeliger, N. \& Ladmiral, J.-R. (2010). De la localisation à la délocalisation - le facteur local en traduction. Meta, 55(4), 615-625.

https://doi.org/10.7202/045681ar d'utilisation que vous pouvez consulter en ligne. 


\title{
De la localisation à la délocalisation - le facteur local en traduction
}

\author{
NICOLAS FROELIGER \\ Université Paris Diderot - Paris 7, Paris, France \\ nf@eila.univ-paris-diderot.fr \\ JEAN-RENÉ LADMIRAL \\ ISIT, Paris, France \\ Université Paris X - Nanterre, Paris, France
}

\begin{abstract}
Il existe des cartes de territoires sur lesquels nous ne pourrons jamais avoir de renseignements exacts. [...] [L]'essentiel est de rester conscient de la signification «multiordinale», c'est-à-dire polyvalente, des mots que l'on entend ou que l'on prononce.
\end{abstract}

(Van Vogt 1957: 178)

Paradoxalement, pour traiter d'une problématique contemporaine comme celle qui fait l'objet thématique du présent numéro de Meta, il se trouve que nous sommes amenés à partir de l'immémorial couple paradigmatique que constituent l'espace et le temps. Pour traduire comme pour poser une réflexion sur la traduction, nous avons en effet besoin de nous situer à l'intérieur d'un cadre: on ne traduit pas, on ne cherche pas à théoriser - et, bien sûr, on n'enseigne pas - dans le vide ou dans l'absolu. Le plus immédiat de ces modes de repérage est sans doute le temps. Il est possible d'y distinguer (au moins) deux aspects.

D'une part, il y a l'histoire de la traduction, avec un axe «spirituel» ou religieux, qu'on pourra faire remonter à la Bible des Septante. On observera alors, à la suite de la rédaction de la Vulgate par saint Jérôme, que s'opère une dialectique entre l'intention «cibliste» de la traduction elle-même et son invocation «sourcière» dans une perspective de la dogmatique ecclésiale (voir le concile de Trente, qui déclare "authentique» et donc intouchable la traduction de Jérôme). Il faut aussi prendre en compte un arc profane («temporel»), avec la naissance et le développement de traductions commerciales et juridiques. Sans oublier, bien sûr, le vaste continent des traductions littéraires, y compris celles des textes de divertissement: ces traductionslà sont beaucoup plus diverses et «libres», mais en principe moins durables. Les unes et les autres étant par ailleurs tributaires d'éléments idéologiques plus ou moins déterminants.

D'autre part, il faut considérer les conditions matérielles d'exercice de la profession et leur évolution. Sans remonter jusqu'à la correspondance diplomatique des potentats locaux du Croissant fertile avec leur suzerain égyptien, rappelons, à cet égard, qu'il y a quelque vingt-cinq ans, un délai de trois semaines - temps d'acheminement par voie 
postale compris - pour traduire vingt pages normalisées était jugé «serré». On a vu des demandeurs proposer spontanément un tarif d'urgence dans de tels cas de figure... C'était l'époque, pour les professionnels les plus sophistiqués, des dictaphones, des rudimentaires machines de traitement de texte, qui ont fait long feu, puis des premiers ordinateurs individuels. Ensuite sont arrivés, successivement, télécopieur, modem, Internet, courrier électronique, traduction assistée par ordinateur, linguistique sur (ou de) corpus, extracteurs de terminologie et bien d'autres outils encore. Tout cela a modifié à la fois la demande, la façon de traduire, les conditions économiques d'exercice, l'organisation des métiers et, par voie de conséquence, la façon de penser la traduction. On mesurera cette évolution à la lumière d'une anecdote remontant à 1984. Une traductrice anglophone établie en France suscitait alors un tollé en proclamant lors d'une réunion professionnelle: "Translators should use typewriters »...

Cette dimension de la temporalité conduit à s'interroger sur les paramètres de la qualité et de l'acceptabilité des traductions. Pourquoi estimait-on légitime de modifier forme, fond et intrigue, par rapport à l'original au XVII ${ }^{\mathrm{e}}$ siècle et pas aujourd'hui (voir Mounin 1955)? Pourquoi certaines façons de traduire sont-elles considérées comme valides à une époque et pas à d'autres - au point que les œuvres qui en sont issues se retrouvent avec le temps bannies du champ de la traduction (comme la paraphrase du monologue de Hamlet par Voltaire ${ }^{1}$ ou la réécriture de Little Women $^{2}$, devenu Les quatre filles du docteur March, une fois adapté par Hetzel ${ }^{3}$; voir en particulier Le Brun 2003)? Du coup, qu'appellera-t-on traduction et traducteurs dans dix, vingt ou cinquante ans? Cette question se posant tout spécialement à ceux qui sont non seulement chercheurs, mais aussi responsables de formations en traduction, et a fortiori aux professionnels eux-mêmes.

Mettre en lumière l'historicité du fait traductionnel est donc un excellent moyen d'aborder ce dernier dans son épaisseur, sa richesse, sa diversité, ses contradictions. Ainsi échapperait-on à une partie des naïvetés si répandues touchant cette activité. Cela conduit néanmoins à opposer très nettement (trop?) les grands textes, qui expriment ce qu'il y a de plus subtil et de plus profond dans l'esprit humain, et puis tous les écrits que l'on range dans le rayon hétéroclite mais bien pratique des écrits pragmatiques, qui sont censés n'avoir qu'une visée de communication. Puisque le critère de qualité essentiel des grands textes est leur vocation à durer, cela n'incite-t-il pas à mépriser les seconds au profit des premiers, comme l'a admirablement synthétisé Henri Meschonnic?

C'est sur les grands textes anciens que s'accumulent les traductions. C'est là qu'on peut confronter un invariant, et ses variations. Leur pourquoi, leur comment. Le seul terrain d'expérimentation du langage: où peuvent indéfiniment recommencer des expériences. Là, traduire est une poétique expérimentale. (Meschonnic 1999: 11)

Le jeu de la temporalité est donc doublement éclairant: d'une part, l'histoire de la traduction éclaire la traductologie et, d'autre part, la question de la pérennité des grands textes mérite d'être creusée, en particulier à l'heure d'Internet. Mais quoi qu'il en soit de cette référence au temps, une réflexion sur la traduction en appelle aussi à la problématique de l'espace.

Pourquoi l'espace? Parce qu'il est tout aussi immédiat dans notre système d'appréhension et de représentation du monde (voir Lakoff et Johnson 1980/2003); parce 
que traduire, dans la vie réelle, cela suppose en premier lieu de se positionner. Il est crucial, en l'occurrence, de pouvoir s'orienter, et donc d'abord de savoir qu'il faut s'orienter, notamment à partir d'une demande et de conditions d'exécution, de façon à produire un texte qui «fonctionne». Un peu comme les architectes parlent d'«apprivoiser la lumière» dans la conception d'un bâtiment: l'architecture comme «le jeu savant, correct et magnifique des volumes sous la lumière» (Le Corbusier 1923/1977: v). Car en traduction comme en architecture, l'opération coïncide avec le résultat.

Le paradigme de l'espace, qui fait la matière du présent recueil, est omniprésent dans la traduction: en technique, dans l'audiovisuel, en littéraire, mais aussi en publicité, dans les diverses modalités juridiques de la traduction, et jusque dans la savoureuse sphère culinaire. Sans parler de la transposition de l'humour, des jeux de mots, de l'ironie, de la dérision... Il y a lieu de considérer ici deux aspects.

Depuis quelques années, on observe d'une part une montée en puissance et une évolution rapide, parmi les métiers de la traduction, de la profession de localisateur (ou «localiseur»). D'autre part, on assiste à une recomposition dans l'espace des activités liées à la traduction: ouverture de nouveaux marchés, apparition et autonomisation de nouveaux métiers, réorganisation des flux économiques sous l'effet de la mondialisation, émergence d'une «ergonomie du poste de travail» en traduction ${ }^{4}$ dans laquelle, avec l'évolution rapide des outils informatiques, le traducteur (humain) se trouve menacé dans sa position centrale, au profit de ce que l'on appelle la station de travail du traducteur (L'Homme 2001 ou Froeliger 2007). À première vue très hétérogènes, ces phénomènes sont en fait étroitement liés, et l'étude du facteur local permettra de comprendre ce qui se passe aujourd'hui et d'anticiper ce qui pourrait se passer demain dans le monde de la traduction. D'où notre titre: «De la localisation à la délocalisation: le facteur local en traduction».

Une telle démarche suppose que soit précisé le concept de localisation - ce qui ne va pas sans difficulté. En effet, les définitions dont nous disposons ne sont pas toujours très éclairantes. Pour la LISA (Localization Industry Standards Association), par exemple, «Localization is the process of modifying products or services to account for differences in distinct markets ${ }^{5} »$. Mais à ce régime-là, on pourrait aller jusqu'à croire que placer le volant d'une automobile à droite ou à gauche relève de la localisation... Quelque peu vague, les termes products et services renvoient en fait, ici, à deux grandes formes de localisation (logicielle et web) et certains auteurs précisent que «la localisation des aides en ligne ou de la documentation relève des mêmes méthodes et compétences que la traduction “classique” " (Martin 2005-2006: 171). Plusieurs contributions à ce recueil reviennent sur ce problème. C'est notamment le cas de Carmelo Cancio et Sydney Belmonte, qui se penchent sur les enjeux de différentes définitions, avant de défendre une vision en plan serré, où la logique de la recherche appliquée plonge ses racines dans une pratique professionnelle. Pour eux, donc, la localisation est bel et bien distincte de la traduction:

[L]a spécificité [de la localisation] est que, à la différence de la traduction, elle ne porte pas sur un document, mais sur un programme, dont la finalité n'est pas de communiquer, mais de faire. (Cancio et Belmonte, ce numéro: 665)

Pour Philippe Lacour et ses coauteurs, au contraire, il s'agit d'un «mouvement beaucoup plus large que la simple déclinaison multilingue des interfaces Web» (Lacour, Bénel et al., ce numéro: 676). En termes simples, on peut considérer que la 
traduction s'arrête et la localisation commence lorsqu'on change de matériau traité: est-ce qu'on travaille sur du texte (traduction) ou d'abord sur du code (localisation)? Mais la simplicité peut être trompeuse: bien souvent, les délimitations se font moins précises, comme c'est d'ailleurs généralement le cas dans les métiers de la traduction, dont une part de l'unité tient précisément au caractère évolutif et donc flou des objets traités. Ces hésitations constituent toute la difficulté et tout l'intérêt d'une réflexion qui part de la localisation. Il y a, d'une part, le risque de parler de choses différentes artificiellement rassemblées sous le même vocable. Il y a, de l'autre, la possibilité de transposer des mécanismes de pensée pour en faire ressortir les points communs et les points de divergence: la traduction et ses métiers sont pluriels. C'est pour honorer cette diversité que nous avons choisi, pour ce volume, de prendre au mot la question du local, c'est-à-dire de la mettre à l'épreuve de multiples domaines et problématiques dans l'espoir d'en éclairer les points de convergence. Il en résulte une diversité pour plusieurs raisons. D’abord, parce que la traduction se déploie dans un système analogique: un traducteur ne cesse de «replier» (comme aurait pu écrire Gilles Deleuze 1988) sur un domaine nouveau ce qu'il a appris, ce qu'il a cru comprendre, ailleurs. Ensuite, parce que ce domaine d'activité, sous l'angle de la recherche, demeure distinct des autres, ce qui soulève un autre problème d'ordre «spatial»: celui du positionnement de la recherche en traduction pragmatique par rapport aux domaines connexes. Pour nous, le facteur local est donc à la fois un thème qui renvoie à des problèmes de traduction bien spécifiques, un principe de distinction et un outil de pensée.

La préoccupation qui a motivé la rédaction de ce volume est bien là: quelle est l'influence du «territoire», c'est-à-dire de la culture et de la civilisation, dans l'activité de traduction? Qu'est-ce qui, dans cette opération, échappe au jeu sur la langue? De quelles manières et avec quelles implications? C'est ici qu'une fois encore l'époque nous oblige à avancer : c'est sous la pression de la mondialisation, des avancées techniques, de la communication quasi instantanée que ces questions s'imposent à nous. Il y a là des faits marquants de notre temps. Ces phénomènes qui font partie de notre quotidien suscitent non seulement des besoins en traduction nouveaux, plus importants et mieux ajustés à leur public cible, mais aussi un éclatement de la fonction de traducteur en une multitude de métiers, en même temps qu'une reconfiguration des marchés à l'échelle internationale: de la localisation à la délocalisation, là encore. Tout cela pour apporter des éléments de réponse à ces questions lancinantes que se posent les traducteurs aujourd'hui dans leurs divers cénacles: en quoi la mondialisation modifie-t-elle ou risque-t-elle de modifier les conditions d'exercice de la profession de traducteur dans un pays donné? Que traduit-on, dans quels lieux, pour quels volumes, à quel tarif, pour quels publics, avec quels incitations ou découragements institutionnels? Le risque existe-t-il d'une migration de certains métiers de la traduction vers des régions où le niveau de vie est moindre, et si oui, comment y faire face? On peut se demander où situer le problème entre la réalité et le fantasme. Il y aurait lieu, aussi, de s'interroger sur le versant institutionnel: quelle est la carte des programmes de formation en traduction aujourd'hui en France, en Europe et dans le monde - avec quels effets sur la constitution et, parfois, la désintégration d'une profession? Que l'on pense à la traduction audiovisuelle...

On l'aura saisi, c'est donc dans ce sens très large (symbolique, diraient d'aucuns) que nous concevons cette problématique, en misant sur l'apport de facettes différentes 
pour enrichir notre vue d'ensemble. Pour cartographier cet ensemble, il convenait de commencer par aborder quelques aspects théoriques, avant d'en venir à différents domaines et applications.

Il s'agit, dans un premier temps, de penser local, localisation et délocalisation. Et c'est d'abord à Carmelo Cancio et Sydney Belmonte («De la localisation à la délocalisation: enjeux professionnels») de se pencher de plus près sur l'évolution de cette profession au fur et à mesure des progrès de la mondialisation. Le premier de ces auteurs allie à sa compétence universitaire une longue expérience de localisateur; le second vient également du monde professionnel et participe à l'aventure du master pro ILTS, à l'Université Paris Diderot. Ils montreront comment on est passé d'une activité orientée vers la satisfaction des besoins des utilisateurs à un éclatement géographique doublé d'une parcellisation des fonctions, qui conduit les rédacteurs à suggérer que localisation et délocalisation sont in fine deux des facettes d'une même réalité. Les corollaires de cette évolution sont une baisse de la qualité des produits concernés, la précarisation de beaucoup de professionnels et la montée en puissance de grandes entreprises de localisation et de traduction.

Qui dit facteur local dit par ailleurs aspects culturels et psycho-relationnels. C'est Jean-René Ladmiral, auteur entre autres choses d'un ouvrage de référence sur la traduction (Ladmiral 1979/1994) et d'un autre sur la communication interculturelle (Ladmiral et Lipiansky 1989), qui posera ensuite cette question, en commençant lui aussi par conceptualiser traduction et localisation. En effet, la traduction est en permanence confrontée à la question de l'interculturel. Mais ce terme à la mode ne tend-il pas à n'être qu'un pur habillage pour conférer un supplément symbolique et un complément de rentabilité à l'activité de traducteur? Ou bien recouvre-t-il quelque chose de réellement nouveau? Comme l'indiquait en 2009 l'appel à communications du colloque Implicites, stéréotypes, imagination, organisé par l'INALCO (Institut national des langues et civilisations orientales, à Paris),

tout système de références culturelles sous-entend une organisation de la réalité, une logique et une vision du monde, des savoirs et des aspects du réel implicites (plus ou moins ostentatoires ou secrets), acceptés et diffusés par les membres d'une communauté donnée. Il s'agit notamment du champ de la connotation, l'« atmosphère affective» des mots, celle qui recouvre tous les sens indirects ${ }^{6}$.

On peut ainsi déboucher sur la question de la retraduction: Pourquoi donc retraduire? Telle était la thématique d'une livraison de la revue Palimpsestes (Bensimon et Coupaye 2004) et d'un colloque à l'Université de Mulhouse fin 2009 (La retraduction: les belles revisitées de la littérature européenne au $\mathrm{xx}^{\mathrm{e}}$ siècle ${ }^{7}$ ). Quels textes retraduirat-on? Et qu'en est-il de la traduisibilité en général? Sans parler de la part qui revient dans la traduction à ces représentations que Cornelius Castoriadis (1975) qualifie «d'imaginaires sociales».

Se pose ensuite la question de l'unité des sciences de la traduction à la lumière des problématiques du local. C'est Nicolas Froeliger qui, ici, thématise «le facteur local comme levier d'une traductologie pragmatique». À partir de quelques exemples empruntés à des textes pragmatiques, il entreprend dans un premier temps de relier ces cas concrets à diverses approches théoriques. Il en vient néanmoins à constater que cela conduit à faire dire, artificiellement, des choses semblables à des auteurs par ailleurs incompatibles. N'y a-t-il pas là une forme d'impasse théorique? D'où la nécessité d'une recomposition qui amènerait à autonomiser la recherche en traduction 
pragmatique par rapport à une traductologie littéraire qui tient souvent le haut $\mathrm{du}$ pavé, y compris parfois sur le plan institutionnel. Ces analyses débouchent sur un trait un peu surprenant, que l'auteur pense décisif, à savoir : c'est lorsqu'il y a défaut (inaperçu) d'identité entre source et cible que l'on peut dire qu'il y a véritablement traduction!

Dans la foulée de ces quelques approches théoriques, on en vient à traiter différents outils, puis à envisager divers domaines d'application, à décentrer la question, et, enfin, à terminer sur l'examen de deux marchés spécifiques de la traduction. C'est d'abord à l'équipe coordonnée par Philippe Lacour, qui comprend Aurélien Bénel, Franck Eyraud, Any Freytas et Diana Zambo, de nous présenter l'ambitieux projet «TraduXio: un laboratoire numérique de translocalisation culturelle». Sous l'éclairage interdisciplinaire de la philosophie, de la linguistique et de l'informatique, la problématique territoriale se déploie ici selon plusieurs axes. D’abord, en établissant des ponts entre des domaines souvent considérés comme séparés: technologies de l'information et de la communication (TIC), d'une part, sciences humaines, d'autre part; ensuite, en ceci que la plateforme qui fait l'objet de leur projet de recherche est "acentrique»: la traduction ne recourt pas ici à une langue pivot, ce qui autorise quelques espoirs en matière de diversité linguistique. Plus généralement, cela tend à montrer comment les modèles intellectuels issus de la traduction peuvent permettre d'informer d'autres secteurs connexes, au point que les auteurs mentionnent des «fonctions de pollinisation (déterritorialisation et reterritorialisation) de la traduction", à l'époque du Web 2.0. Avec en filigrane la promotion de nouveaux biens communs, grâce à la constitution de communautés virtuelles de traducteurs.

Il convient ensuite de se pencher sur la façon dont se décline le facteur local dans trois domaines bien délimités. Parler de territoire, c'est logiquement parler de droit, s'interroger sur les conditions de fonctionnement d'un marché et poser la question de l'exercice de la souveraineté. Le paramètre de la performativité, déjà évoqué par Carmelo Cancio et Sydney Belmonte se présente ici d'une tout autre manière. Avocate et doctorante à l'Université Paris 10 Nanterre, Sylvie Monjean-Decaudin traite ainsi de la «territorialité et de l'extraterritorialité de la traduction du droit», en se penchant sur deux formes d'équivalence traductionnelle, qu'elle qualifie respectivement d'horizontale et de verticale. La première est uniformisante: c'est celle du droit international ou régional négocié, qui prendra ensuite la forme de différentes versions linguistiques. La seconde est au contraire respectueuse des caractéristiques culturelles et a pour but la connaissance du droit des autres, mais aussi l'administration de la justice. Avec néanmoins toujours le risque qu'une hégémonie linguistique, juridique et/ou territoriale vienne perturber le fragile équilibre qui permet de se tenir à distance de deux excès: trop de référent culturel (traduction territorialisée) ou trop peu (traduction non territorialisée).

Nolwenn Mingant, enseignante chercheuse à l'Université Sorbonne NouvelleParis 3 et spécialiste des questions de cinéma, propose ensuite l'analyse d'un film que son réalisateur avait, lors du Festival de Cannes 2009, annoncé comme impossible à doubler ${ }^{8}$ : Inglourious Basterds, de Quentin Tarantino. Pourquoi impossible? Dans une certaine mesure parce que les accents régionaux, en allemand et en anglais, y jouent un rôle considérable, mais surtout parce que les témoins et bientôt victimes de la première scène (une famille juive cachée par un agriculteur français sous son plancher) de ce long métrage repose sur le fait qu'une partie des personnages ne 
comprennent pas la conversation (en anglais) des deux protagonistes: l'agriculteur en question et un officier SS. L'irréalisme linguistique complet de cet incipit constitue au demeurant une des marques poétiques revendiquées de ce film. Cette contribution envisage alors les différentes stratégies adoptées pour le doublage de ce film - car il a toutefois bien eu lieu - et qui ont pour effet de réduire la place attribuée à la langue par le réalisateur dans la construction narrative et thématique de son film. Cet article pose donc la question de l'articulation entre traduction, sous-titrage et doublage, problématique qui gagne en importance à l'heure où se multiplient les films multilingues, aux États-Unis et ailleurs.

Autre interrogation: les attentes d'un public sont-elles les mêmes dans des pays différents? Poser cette question, c'est déjà y répondre. Reste à savoir ce que cela signifie concrètement pour le traducteur, mais aussi pour le lecteur. Fréquemment traitée dans l'étude des traductions littéraires, cette problématique se rencontre tout autant, avec des modalités différentes, dans un genre que la recherche traductologique ne prend que très rarement en compte, à savoir la culture de masse. Sous le titre " "Tout un monde d'évasion": Adapter les romans sentimentaux pour un lectorat français ", Maïca Sanconie, traductrice littéraire, romancière et enseignante à l'Université d'Avignon et des Pays du Vaucluse, se penche sur l'inépuisable et frémissant sujet des romans sentimentaux à caractère historique. Elle nous montre comment la narration a pour but de susciter un espace décroché de l'univers familier des lectrices et destiné à capter leur imagination. À l'inverse de la traduction pragmatique, forcément référée au réel (Froeliger 2004), ne pourrait-on pas dire que la traduction se donne ici pour objectif non pas une stratégie d'adaptation au référent, à la norme et au local mais «la représentation d'un exotisme sans faille». Il s'agit donc de soutenir, et même de renforcer, les fantasmes susceptibles de surgir du récit.

$\mathrm{Au}$-delà de ces approches thématiques, il y a lieu de revenir sur la relation «topologique» existant entre traduction et domaines connexes. Après l'articulation entre traduction et localisation, qu'en est-il des rapports entre traduction et rédaction/ communication technique? C'est le sujet, en anglais, de Patricia Minacori et Lucy Veisblat, toutes deux membres de l'Université Paris Diderot: "Translation and technical communication: chicken or egg?» On sait qu'aux États-Unis, ces deux professions ont toujours été distinctes. Il n'en va pas de même en France, où la rédaction technique (devenue entre-temps communication technique) s'est développée à partir de la traduction, avant de commencer à s'en détacher. Nos auteures reviennent donc sur l'historique de cette autonomisation et sur les relations entre les deux spécialités au sein des métiers de la traduction. Elles proposent dans un deuxième temps l'élaboration d'un cadre de référence pour la formation des communicateurs techniques, en s'inspirant de la liste des compétences établie dans le cadre du projet européen EMT (European Master's in Translation ${ }^{9}$ ), qui tend à constituer au demeurant un fait marquant sur le Vieux Continent, non seulement dans l'organisation des formations aux métiers de la traduction, mais également pour les marchés qui se situent en aval de ces formations et pour ceux qui y travaillent.

Tout le monde sait, par ailleurs, qu'il n'existe pas de bonne traduction sans terminologie précise. Mais que faire, lorsque les concordances terminologiques ne sont que partielles d'une langue à une autre? C'est sur ces passionnantes interrogations que travaillent depuis quelques années Pierre Avenas, ancien ingénieur, et Henriette Walter, linguiste de renom et professeure émérite à l'Université Rennes 2 de Haute 
Bretagne. Ensemble, ils sont les auteurs de plusieurs ouvrages fourmillant de zébus, émeus et autres pangolins, sur les noms d'animaux (2003,2007, avec un troisième en préparation à l'heure où nous écrivons ces lignes). Dans cette profession saturée de paradoxes, la question pour le traducteur est là encore: à quel moment peut-on et doit-on se permettre de ne pas être exact? En effet, les noms vernaculaires, avec leur cortège d'idiomatismes et de variantes régionales, suivent des dynamiques bien plus complexes que celle des taxinomies scientifiques; et l'on retrouve bien sûr le jeu du couple infernal dénotation/connotation dès lors qu'on s'aventure sur les terres du mythe, de la littérature ou de l'œuvre artistique en général. Ce qui conduit à plaider pour une approche diversifiée associant connaissances relevant des sciences naturelles et considérations linguistiques.

À l'heure où nous voilà bien avancés sur le chemin de la déterritorialisation, pour reprendre un terme cher à Gilles Deleuze et Félix Guattari (1980), Julie Noirot, de l'Université Paris 8, mettra ensuite pour ainsi dire la question du territoire sur sa tête, à propos de «La photographie d'architecture, un art de la traduction?». Il ne s'agit plus, cette fois, de cartographier les voisinages entre la traduction et ses voisins, mais d'emprunter certaines théories de la traduction et de l'interprétation pour tenter d'appréhender un domaine a priori bien spécifique, ce qui nécessite un travail de définition et une délimitation des enjeux. L'auteure nous montrera comment certains concepts, certaines notions, voire certaines méthodes traductologiques peuvent s'appliquer à la photographie d'art, en reprenant notamment le couple conceptuel "sourciers/ciblistes", et avec pour effet d'interroger in fine le statut du photographeinterprète. Ce sera également l'occasion pour chacun de se souvenir que Walter Benjamin n'est pas seulement l'auteur de sa fameuse étude métaphysique sur La tâche du traducteur (1923).

Toujours dans la problématique de la localisation et de la délocalisation, les deux dernières contributions ont pour objet la traduction et ses avatars en tant que phénomènes localisés. Y compris à l'heure de la mondialisation, qui vient évidemment bouleverser beaucoup de choses, les marchés n'étant pas organisés de la même manière dans un pays et dans un autre. Nous aurions pu, pour ce dernier ensemble, parler de la Norvège, seul pays à notre connaissance dans lequel une grève générale des traducteurs a non seulement été possible, mais a en outre débouché sur une renégociation globale des conditions d'exercice entre les professionnels et les donneurs d'ordre ${ }^{10}$. Nous aurions pu envisager le marché canadien, où l'obligation légale de bilinguisme est le gage à la fois d'un volume de traduction très conséquent et du pacte social entre les deux principales communautés linguistiques. Nous aurions pu examiner le cas extrêmement intéressant (au Royaume-Uni, par exemple) de ce qu'on appelle en anglais community interpreting, dont l'équivalent français, visant à la synthèse entre traduction et interprétation interculturelles, est encore hésitant. Nous avons préféré mettre à l'honneur deux ensembles géographiques dont il est moins fréquemment question, et sans doute à tort.

Anca Greere, maître de conférences à l'Université Babes-Bolyai de Cluj-Napoca, en Roumanie, décrit comment le marché de la traduction s'y est réorganisé depuis le changement de système politique (et les nouvelles alliances géopolitiques) survenu en 1989, et en particulier avec le processus d'accession de ce pays à l'Union européenne en 2007 (Translation in Romania: Steps towards recognition and professionalization). Partant d'une situation dans laquelle le russe était langue dominante et où les seuls 
programmes de traduction concernaient le littéraire, les pouvoirs publics, les universités et les professionnels ont conjugué leurs efforts pour mettre en place en un temps remarquablement bref un marché digne de ce nom, réorienté vers les nouveaux partenaires économiques de la Roumanie et aujourd'hui de mieux en mieux reconnu à l'échelle européenne et mondiale. Cette étude s'appuie sur une imposante documentation pour revenir sur la prise en compte des problématiques de la traduction, la reconnaissance socioéconomique des métiers des langues et la formation d'une conscience professionnelle collective, envisagée comme un marqueur de professionnalisation.

Autre marché en devenir - et ô combien vaste! -, l'Afrique subsaharienne. Jeannine Gerbault, maître de conférences à l'Université Michel de Montaigne, Bordeaux 3, s'attelle à la lourde tâche de clore ce numéro thématique sur ce sujet: «Localisation, traduction et diversité sociolinguistique en Afrique subsaharienne: stratégies et perspectives». L'ensemble géographique considéré concentre à la fois difficultés et perspectives d'avenir. Se posent plusieurs problèmes: multiplicité des langues et des systèmes d'écriture d'origine locale, dont les aires géographiques ne correspondent qu'occasionnellement aux découpages des frontières; superposition à ce premier ensemble de langues véhiculaires originaires d'Afrique ou d'autres continents; fragilité des infrastructures, contrebalancée par un développement très rapide des TIC. En centrant son analyse sur ces technologies de l'information et de la communication, l'auteure dresse un tableau large, et là aussi fort solidement documenté, de la manière dont s'organisent la traduction et la localisation dans cette zone, sous la double influence de la mondialisation et des politiques qui visent à promouvoir la diversité linguistique - non d'ailleurs sans difficulté.

Nous irons donc du général au particulier: localisation stricto sensu, voisinages théoriques, aspects psychologiques, lexicologie, culture de masse, esthétique, logique du marché et politiques de la traduction : la fonction de la traduction - et la réflexion sur cette dernière - consiste à prendre en compte tout cet ensemble sur le plan pratique, et donc à une échelle modeste et discrète. Il s'agit de montrer comment la pluralité des approches qui sont présentées dans ce numéro débouche sur une forme de cohérence placée sous le dénominateur commun de la traduction, tout en s'attachant à mettre en abyme les faces d'un même ensemble que constituent la pratique et la recherche. Car dans ce panorama, nous répétons que le critère de la pertinence, c'est le destinataire qui le fixe. Ce qui pose la question de savoir à qui s'adresse la recherche. Pour nous, la recherche s'adresse aux enseignants et chercheurs, certes, mais aussi - et, il nous arrive de le penser, en premier lieu - aux professionnels, d'aujourd'hui et de demain. Il s'agit de construire des passerelles entre ces différents publics, afin que chacun s'en trouve enrichi. C'est dans cette logique qu'un certain nombre d'articles du présent numéro thématique ont fait d'abord l'objet de présentations lors de la troisième Journée de la traductologie de plein champ, organisée le 12 septembre 2009 à l'Université Paris Diderot ${ }^{11}$, avant d'être repris et approfondis pour cette publication.

Il nous a donc semblé expédient d'associer aux chercheurs des professionnels en activité. S'agissant des premiers, nous avons à la fois des programmes de recherche qui n'en sont encore qu'à leurs débuts, comme celui que présente l'équipe regroupée autour de Philippe Lacour, et d'autres qui sont déjà achevés, comme celui dont JeanRené Ladmiral esquisse ici un bilan. Quant à la thématique de la localisation elle- 
même, on pouvait l'envisager de deux façons. Soit on se saisissait du concept au sens restreint qu'il a pris, au cours des années 1980, dans le domaine informatique: et il n'y aurait peut-être pas eu la matière de tout un numéro de Meta; et surtout, ce dernier aurait pu sembler excessivement «technique». Soit on entendait la localisation au sens large, comme nous l'avons fait, et cela ouvrait à un domaine illimité. Entre le trop peu et le trop, nous avons choisi de procéder par «carottage», en renonçant à couvrir l'ensemble du champ d'étude possible. D’où aussi la diversité des contributions qui a déjà été indiquée plus haut. Encore une fois: la carte n'est pas le territoire, comme l'a magistralement illustré le grand Borges, et comme ont pu le reprendre un Korzybski ${ }^{12}$ dans le cadre de sa Sémantique générale, ou un Van $\operatorname{Vog} t^{13}$ (1957), auquel nous avons emprunté l'épigraphe de la présente introduction, ce qui nous était une façon de rendre hommage entre les lignes à son traducteur, Boris Vian. Et qui pourra ignorer que la rentrée littéraire française 2010 nous aura apporté un très attendu $\mathrm{La}$ carte et le territoire signé Michel Houellebecq ${ }^{14}$ ? À travers ces préoccupations d'aujourd'hui et de toujours, nous pensons ainsi proposer à nos lecteurs non pas un cadre rigide, mais bien des moyens pour s'orienter dans la jungle de ces métiers de la traduction en perpétuel devenir.

\section{REMERCIEMENTS}

Nous souhaitons exprimer toute notre gratitude aux membres (anonymes) de nos deux comités de lecture: celui mis en place par les responsables de ce numéro thématique et, en aval, celui de Meta, pour la pertinence et la précision de leurs remarques, ainsi qu'à tous les auteurs de ce numéro et aussi - et nous serions tentés d'écrire en particulier - aux professionnels, chercheurs, enseignants et étudiants qui font vivre la Traductologie de plein champ.

\section{NOTES}

1. Voltaire (François-Marie Arouet, dit) (1733): Lettre XVIII. Sur la tragédie. Lettres philosophiques. $<$ http://www.voltaire-integral.com/Html/22/11_Lettre_18.html>. Consultée le 25 août 2010.

2. Alcotт, Louisa May (1880): Little Women; or Meg, Jo, Beth, and Amy. Cambridge (Massachusetts): John Wilson and Son.

3. Alcotт, Louisa May (1880): Les quatre filles du Docteur March. (Adaptation de Pierre-Jules StAHL [pseudonyme de Pierre-Jules Hetzel]). Paris, Hachette, «Idéal-Bibliothèque».

4. Voir à ce sujet le colloque Traduction et ergonomie, organisé par Élisabeth Lavault à Grenoble, les 16 et 17 octobre 2010: <http://calenda.revues.org/nouvelle16531.html>. Consulté le 20 juillet 2010. Mentionnons également le colloque qui s'est tenu à Mons en 1991 : voir Clas et Safar (1992).

5. Disponible sur le site de la LISA : <http://www.lisa.org/Localization.61.0.html>. Consulté le 22 juin 2010 .

6. Journées d'études de l'INALCO, laboratoire PLIDAM, les 11 et 12 juin 2009. Programme disponible à l'adresse <inalco-front1.heb.fr.colt.net/...Programme11_12juin09_tzende_PG.pdf>. Consultée le 22 août 2010.

7. «Traduction comme moyen de communication interculturelle. Questions de socio-pragmatique du discours interculturel», $\mathrm{XV}^{\mathrm{e}}$ rencontre du Réseau thématique international de recherche et de formation à la recherche Université de Mulhouse, 2-5 décembre 2009.

8. Cité dans l'article «Tarantino enrôle Brad Pitt», Première, n 390. Paris, août 2009.

9. Voir par exemple <ec.europa.eu/dgs/translation/programmes/emt/network/emt_faq_fr.pdf >. Consultée le 24 août 2010.

10. Nous tenons cette information d'échanges avec des traducteurs et des chercheurs, lors de la troisième Journée de la traductologie de plein champ (à Paris, le 12 septembre 2010). Consultée le 16 août 2010, <http://www.eila.univ-paris-diderot.fr/recherche/conf/ciel/traductologie-pleinchamp/3eme_edition>.

11. <http://www.eila.univ-paris-diderot.fr/recherche/conf/ciel/traductologie-plein-champ/index>. Consultée le 17 août 2010. 
12. Korzybsкi, Alfred (1998). Une carte n'est pas le territoire - Prolégomènes aux systèmes non-aristotéliciens et à la Sémantique générale. (Traduit de l’anglais par Didier KoHN, Mireille DE Moura et Jean-Claude Dernis) Collection premiers secours. Paris: Éditions de l'Éclat.

13. Van Vogt, Alfred E. (1957): Les joueurs $d u \bar{A}$. (Traduit de l'anglais par Boris Vian). Paris: Gallimard.

14. HouellebecQ, Michel (2010): La carte et le territoire. Paris: Flammarion.

\section{RÉFÉRENCES}

Benjamin, Walter (1923): Walter Benjamin: Die Aufgabe des Übersetzers. In: Charles BAudeLAIRE, Tableaux parisiens. Heidelberg: Verlag von Richard Weißbach. Consultée le 24 août 2010, <http://www.uni-weimar.de/medien/europa/lehre/ws0405/vatersprache_content/ benjamin1.pdf $>$.

Bensimon, Paul et Coupaye, Didier, dir. (2004): Pourquoi donc retraduire? Palimpsestes. 15.

CAstoriadis, Cornelius (1975): L’institution imaginaire de la société. Paris: Seuil.

Clas, André et SAfar, Hayssam, dir. (1992): L'environnement traductionnel - La station de travail du traducteur de l'an 2001. Montréal: Presses de l'Université du Québec.

Deleuze, Gilles et Guattari, Félix (1980): Mille Plateaux. Paris: Minuit.

Deleuze, Gilles (1988): Le Pli - Leibniz et le baroque. Paris: Minuit.

Froeliger, Nicolas (2004): Felix Culpa: congruence et neutralité dans la traduction des textes de réalité. Meta. 49(2):236-246.

Froeliger, Nicolas (2007): Pourquoi avoir peur de l'informatisation en traduction? Tribune internationale des langues vivantes. 43:40-54.

L’Номме, Marie-Claude (2001): Initiation à la traductique. Montréal: Linguatech.

LADMIRAL, Jean-René (1979/1994) : Traduire: théorèmes pour la traduction. Paris: Payot pour la première édition. Réédité en 1994 et 2010. Paris: Gallimard.

Ladmiral, Jean-René et Lipiansky, Edmond Marc (1989): La Communication interculturelle. Paris: Armand Colin.

Lakoff, George et Johnson, Mark (1980/2003): Metaphors We Live By. Chicago : The University of Chicago Press.

Le Brun, Claire (2003): De Little Women de Louisa May Alcott aux Quatre filles du docteur March: les traductions françaises d'un roman de formation au féminin. Meta. 48(1-2):4767.

Le Corbusier (Charles-Edouard Jeanneret, dit) (1923/1977): Vers une architecture. Paris: Flammarion.

Martin, Camille (2005-2006): Les modèles technologiques de la localisation. In: Daniel GouAdec, dir. Traduction - localisation: technologies et formation. Paris: La maison du dictionnaire, 171-176.

Meschonnic, Henri (1999): Poétique du traduire. Lagrasse: Verdier.

Mounin, Georges (1955): Les belles infidèles. Paris: Éditions des cahiers du Sud.

Mounin, Georges (1963): Les problèmes théoriques de la traduction. Paris: Gallimard.

Walter, Henriette et Avenas, Pierre (2003): L'étonnante histoire des noms des mammifères... de la musaraigne étrusque à la baleine bleue. Paris: Robert Laffont.

Walter, Henriette et Avenas, Pierre (2007): La mystérieuse histoire des noms des oiseaux. Du minuscule roitelet à l'albatros géant. Paris: Robert Laffont. 